\title{
Traitement Des Phosphates Naturels De Tahoua (Niger) Par Quelques Acides Conventionnels
}

\author{
Ousmane Mahamane Sani, (PhD) \\ Laboratoire Matériaux-Eau-Environnement (LAMEE), Faculté des Sciences \\ et Technique, Université Abdou Moumouni de Niamey, Niger \\ Faculté des Sciences et Technique, Université d‘Agadez, Niger \\ Zanguina Adamou, (M.C) \\ Natatou Ibrahim, $(\mathrm{Pr})$ \\ Laboratoire Matériaux-Eau-Environnement (LAMEE), Faculté des Sciences \\ et Technique, Université Abdou Moumouni de Niamey, Niger
}

doi: 10.19044/esj.2017.v13n24p176 URL:http://dx.doi.org/10.19044/esj.2017.v13n24p176

\begin{abstract}
Within the framework of upgrading natural phosphate of Tahoua, a study was carried out on the dissolution of ore by inorganic acid (sulfuric acid, nitric acid) and organic acid (citric acid) solutions according to $\mathrm{pH}$ and attack time. The obtained results show a sudden drop of dissolution rate at $\mathrm{pH}$ above 2 and a dissolution almost zero beyond $\mathrm{pH} 4$. The kinetic study shows fast dissolution of ore one hour after the attack. However, this dissolution is slow beyond one hour with few dissolved phosphorus during a relatively high period of time. Furthermore, the sulfuric acid solutions dissolve the ore better than those of citric and nitric acid: $41.07 \% ; 37.03 \%$ and $35.64 \%$ of dissolved phosphorus respectively for sulfuric, citric and nitric acid solutions at the concentration of $1 \mathrm{~mol} / \mathrm{L}$.
\end{abstract}

Keywords: Citric acid, dissolution, natural phosphate, nitric acid, sulfuric acid

\section{Résumé}

Dans le cadre de la valorisation des phosphates naturels de Tahoua, il a été entrepris une étude de la dissolution du minerai par les solutions d'acides minéraux (acide sulfurique et acide nitrique) et organique (acide citrique) en fonction du $\mathrm{pH}$ et du temps d'attaque. Les résultats obtenus montrent une chute brutale du taux de dissolution au $\mathrm{pH}$ supérieur à 2 et une dissolution quasi nulle du minerai au-delà de $\mathrm{pH}$ égal à 4. L'étude cinétique montre que la dissolution du minerai est rapide au bout d'une heure d'attaque. Cependant elle est lente au-delà d'une heure avec très peu de 
phosphore dissous pendant un temps relativement élevé. Par ailleurs les solutions d'acide sulfurique dissolvent mieux le minerai devant celles d'acides citrique et nitrique : $41,07 \% ; 37,03 \%$ et $35,64 \%$ du phosphore dissous respectivement pour l'acide sulfurique, citrique et nitrique à la concentration de $1 \mathrm{~mol} / \mathrm{L}$.

Mots clés: acide citrique, acide nitrique, acide sulfurique, dissolution, phosphates naturels

\section{Introduction}

La dissolution des phosphates naturels est la propriété donnant le plus d'information sur leur efficacité agronomique car elle conditionne la mise à disposition des ions ortho-phosphates aux plantes. C'est pourquoi de nombreuses recherches sur la dissolution des phosphates calciques naturels ou de synthèse ont été effectuées (Eremia-Georgescu, 2004 ; Lukas, 2008 ; Adel, 2012). Le phosphate naturel de Tahoua a fait l'objet d'un certain nombre de travaux notamment dans la recherche des voies pour son application effective dans le domaine de l'agriculture (Moustapha, 2003 ; André, 1998 ; Natatou, 2004). Du point de vue agronomique, il faut signaler que son application directe comme engrais s'est avérée peu concluante notamment à cause de son caractère pulvérulent (Zanguina, 2011). Toutefois, certaines propriétés comme la forte teneur en pentoxyde de phosphore, la faible présence de métaux lourds peuvent faire du phosphate de Tahoua un produit de grande valeur agronomique.

Dans le cadre de la recherche sur la transformation du phosphate apatitique du Niger pour rendre son phosphore bio-disponible pour les plantes, une étude de la dissolution du minerai par les acides minéraux (acide sulfurique et nitrique) et par l'acide citrique est réalisée dans ce travail. L'acide citrique étant un triacide organique utilisé pour tester la solubilité des phosphates naturels (FAO, 2004) et les acides sulfurique et nitrique étant des acides de référence pour la fabrication des intrants phosphatés. L'objectif visé est de permettre de mieux comprendre la réactivité de la roche phosphatée et de suggérer ainsi des voies possibles d'application notamment celle de la fabrication des engrais phosphatés hydrosolubles.

\section{Matériels et méthodes \\ Matériels}

\section{Le minerai de phosphate}

Le minerai de phosphate utilisé au cours de cette étude est celui de Tahoua au Niger dont le gisement a été identifié dans les années 80 par l'Office National de Recherche Minière du Niger (Natatou, 2005). Les échantillons de laboratoire ont été prélevés sur le minerai brut après un 
broyage et un tamisage fin séparant la matière phosphatée des impuretés constituées en majorité de l'argile et de la gangue siliceuse. L'analyse chimique effectuée au moyen d'un spectromètre Varian liberty 220 ICP/AES a permis d'établir la composition chimique du produit (Natatou, 2005). Les résultats obtenus (Tableau 1) révèlent la présence de calcium, de la silice, des oxydes de métaux et des très faibles teneurs en métaux lourds comme le plomb et le cadmium. La caractérisation physico-chimique du produit a permis de montrer qu'il s'agit d'un phosphate apatitique sédimentaire de type nodulaire (Natatou, 2005).

\section{Les solutions chimiques utilisées}

Les solutions chimiques utilisées au cours de ce travail sont d'origine commerciale. Leurs caractéristiques sont données au Tableau 2.

\section{Appareillage}

Un spectrophotomètre d'absorption moléculaire pour le dosage d'anhydride phosphorique dissous et une balance analytique pour la mesure des masses sont utilisés au cours de ce travail. Leurs caractéristiques sont indiquées au Tableau 3.

Tableau 1 : Composition chimique des phosphates naturels de Tahoua (Natatou, 2005)

\begin{tabular}{|c|c|c|c|}
\hline Eléments majeurs & $\%$ & Eléments traces & $\%$ \\
\hline $\mathrm{CaO}$ & 43,12 & $\mathrm{Ti}$ & 0,050 \\
\hline $\mathrm{P}_{2} \mathrm{O}_{5}$ & 31,24 & $\mathrm{Nd}$ & 0,043 \\
\hline $\mathrm{Fe}_{2} \mathrm{O}_{3}$ & 8,68 & $\mathrm{Sr}$ & 0,043 \\
\hline $\mathrm{Al}_{2} \mathrm{O}_{3}$ & 1,84 & $\mathrm{Zn}$ & 0,014 \\
\hline $\mathrm{Na}_{2} \mathrm{O}$ & 0,26 & As & 0,007 \\
\hline $\mathrm{K}_{2} \mathrm{O}$ & 0,12 & $\mathrm{Ni}$ & 0,006 \\
\hline $\mathrm{Y}_{2} \mathrm{O}_{3}$ & 0,16 & $\mathrm{U}$ & 0,003 \\
\hline $\mathrm{MgO}$ & 0,20 & $\mathrm{Ba}$ & 0,002 \\
\hline $\mathrm{MnO}$ & 0,10 & $\mathrm{Cr}$ & 0,002 \\
\hline \multirow[t]{3}{*}{$\mathrm{Si}_{2} \mathrm{O}$} & 8,00 & $\mathrm{~Pb}$ & 0,008 \\
\hline & & $\mathrm{Cu}$ & 0,005 \\
\hline & & $\mathrm{Cd}$ & 0,003 \\
\hline
\end{tabular}

Tableau 2 : Caractéristiques des produits utilisés

\begin{tabular}{|c|c|c|c|c|}
\hline Produits & $\begin{array}{c}\text { Masse molaire } \\
(\mathrm{g} / \mathrm{mol})\end{array}$ & Densité & Pureté & Origine \\
\hline Acide sulfurique & 98 & 1,84 & $98 \%$ & Normapur \\
\hline Acide nitrique & 63,01 & 1,33 & 52,5 & Normapur \\
\hline Acide citrique & 192,124 & 1,66 & $99 \%$ & Normapur \\
\hline $\begin{array}{c}\text { Tartrate double d'antimoine } \\
\text { et de potassium }\end{array}$ & 333,93 & - & $99 \%$ & Acros Organics \\
\hline $\begin{array}{c}\text { Molybdate d'ammonium } \\
{\left[\left(\mathrm{NH}_{4}\right)_{6} \mathrm{Mo}_{7} \mathrm{O}_{\left.24.4 \mathrm{H}_{2} \mathrm{O}\right]}\right.}\end{array}$ & 1235,86 & - & $99 \%$ & Acros Organics \\
\hline Acide ascorbique & 176,13 & - & $99,7 \%$ & Normapur \\
\hline Phosphate monopotassique & 136,1 & - & $99 \%$ & Sigma-Aldrich \\
\hline
\end{tabular}


Tableau 3 : Caractéristiques des appareils utilisés

\begin{tabular}{|c|c|c|c|}
\hline Appareils & Modèle & Producteur & Pays d'origine \\
\hline Balance & Precisa 205 A & SWISS Quality & Switzerland \\
\hline Spectrophotomètre & Model 4101 & Zuzi Spectrophotometer & Espagne \\
\hline
\end{tabular}

\section{Méthodes}

\section{Attaque des phosphates par les différentes solutions acides}

Une masse de $0,1 \mathrm{~g}$ (granulométrie $<125 \mu \mathrm{m}) \mathrm{du}$ minerai pesée à l'aide d'une balance de précision est introduite dans des béchers de $200 \mathrm{~mL}$ préalablement rincés à l'eau déminéralisée. Ensuite $100 \mathrm{~mL}$ de la solution acide ont été ajouté à chaque échantillon puis le mélange est agité à 500 tours $\cdot \min ^{-1}$ pendant des temps d'attaques différents. A la fin de l'agitation, le mélange est filtré en vue d'un dosage du taux d'anhydride phosphorique $\left(\mathrm{P}_{2} \mathrm{O}_{5}\right)$ dissous.

\section{Préparation de la solution complexante}

La complexation des ions phosphates dissous au cours de l'attaque a été réalisée à l'aide d'une solution complexante. La méthode utilisée pour la préparation de cette solution est celle de Dabin modifiée [11]. Ainsi une masse de 1,056 g d'acide ascorbique est dissoute dans $200 \mathrm{~mL}$ de réactif préparé à partir d'un mélange contenant $12 \mathrm{~g}$ de molybdate d'ammonium, 0,2908 $\mathrm{g}$ de tartrate d'antimoine et de potassium dissous dans $1 \mathrm{~L}$ d'eau déminéralisée et $1 \mathrm{~L}$ d'acide sulfurique $1 \mathrm{~N}$. Ce réactif se prépare à chaque usage.

\section{Détermination du taux du phosphore dissous}

En milieu acide et en présence de molybdate d'ammonium les orthophosphates donnent un complexe phosphomolybdate qui réduit par l'acide ascorbique et développe une coloration bleue, susceptible d'un dosage colorimétrique. L'intensité de la coloration est proportionnelle à la quantité du phosphore dissous dans le milieu réactionnel et est accélérée par le tartrate double d'antimoine et de potassium. Ainsi, on introduit dans une fiole jaugée de $50 \mathrm{~mL}, 5 \mathrm{~mL}$ du filtrat, $8 \mathrm{~mL}$ de la solution complexante puis on fait le volume avec de l'eau déminéralisée. Au bout de 10 minutes, le dosage s'effectue à l'aide d'un spectrophotomètre d'absorption moléculaire (de marque Zuzi 4101) à la longueur d'onde de $860 \mathrm{~nm}$ selon le protocole de Dabin modifié (Mathieu, 2003). Pour une meilleure comparaison des résultats, les solutions étalons ainsi que les solutions des échantillons ont été étudiés dans les mêmes conditions. 


\section{Résultats et discussion}

\section{Dissolution du minerai par les solutions d'acide nitrique}

Tout d'abord l'étude de la dissolution du minerai par les solutions d'acide nitrique a été réalisée à $\mathrm{pH}$ variables. Les résultats de cette étude sont indiqués dans la figure 1 . On remarque que lorsque le $\mathrm{pH}$ est inférieur à 2 , la dissolution est importante avec plus de $35 \%$ de phosphore dissous pour un temps de dissolution de 7 heures. Entre pH 2 et 4 le taux du phosphore dissous chute brutalement avec un point d'inflexion et un palier horizontal au-delà de $\mathrm{pH}$ égal à 4 . Ce palier correspond à une dissolution presque nulle du minerai. Ces résultats corroborent ceux obtenus lors de la dissolution des phosphates naturels de Hahotoé au Togo par les solutions d'acide chlorhydrique (Moursalou K, Zanguina A, 2010).

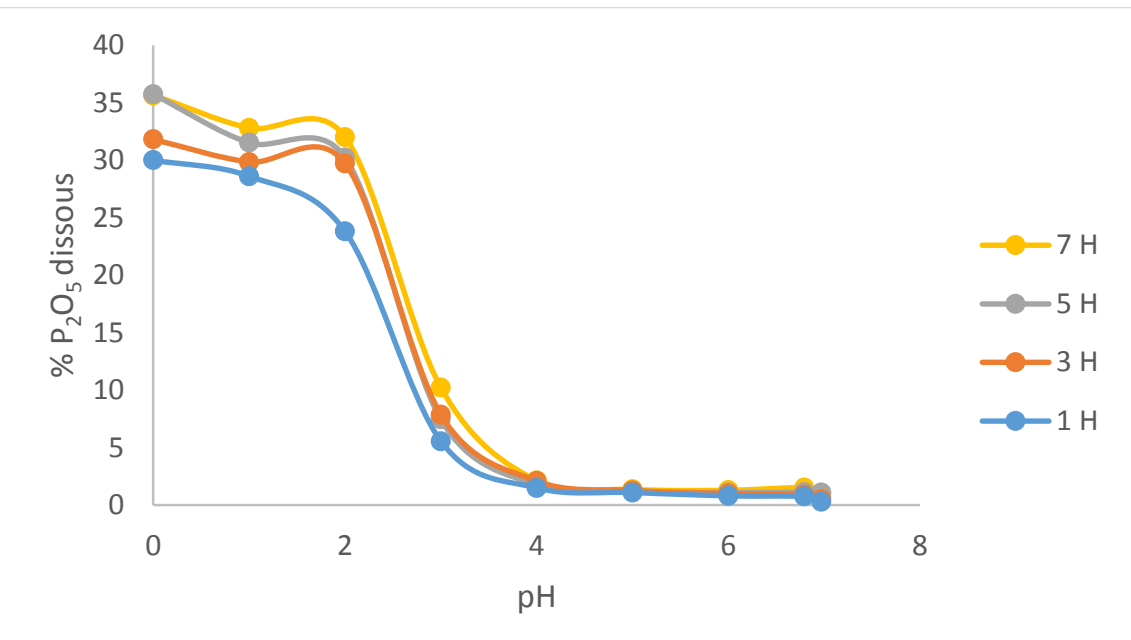

Figure 1 : Taux de phosphore dissous par les solutions d'acide nitrique en fonction du pH

\section{Dissolution du minerai par les solutions d'acide citrique}

Nous avons également mené l'étude de la dissolution du minerai à $\mathrm{pH}$ variables dans les solutions d'acide citrique. La courbe de dissolution (Figure 2) présente la même allure que celle obtenue lors de l'attaque du minerai par les solutions d'acide nitrique. Le taux de phosphore dissous atteint $37 \%$ au pH inférieur à 2 pour un temps de dissolution de 7 heures. Ce taux diminue très rapidement et présente un palier horizontal au-delà de $\mathrm{pH}$ 4. De plus les courbes de dissolution obtenues ont une allure identique pour toutes les durées d'attaque. 


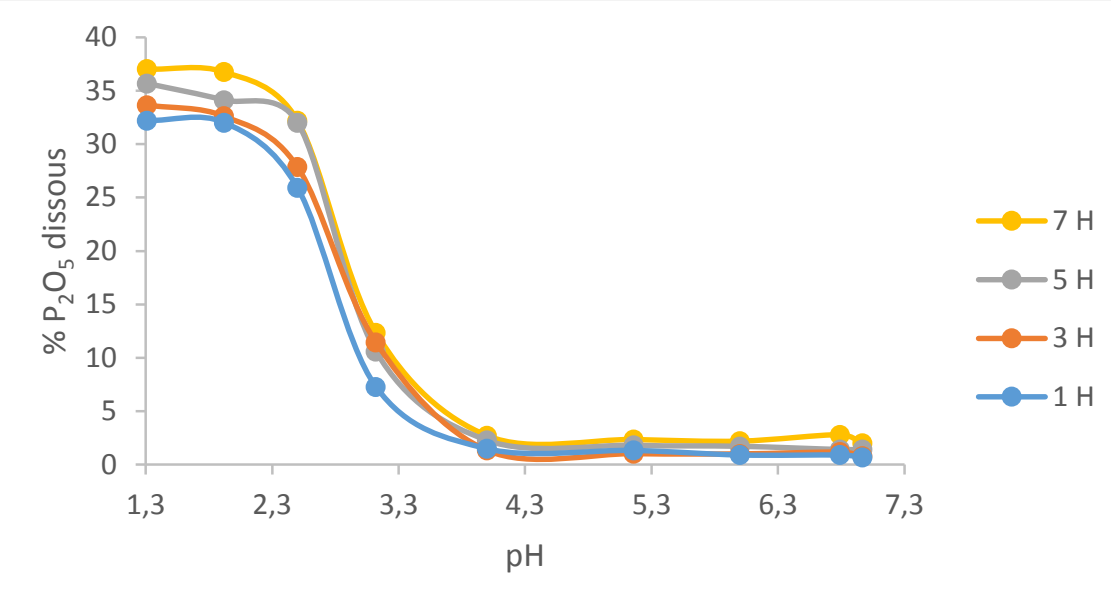

Figure 2 : Taux de phosphore dissous par les solutions d'acide citrique en fonction du pH

Les résultats ci-dessus montrent des comportements identiques des deux acides de référence. La dissolution du minerai est importante en milieu fortement acide dans l'acide minéral $\left(\mathrm{HNO}_{3}\right)$ mais aussi dans l'acide organique (acide citrique). Elle est quasi nulle en milieu faiblement acide. Le pouvoir de solubilisation des phosphates dans les acides minéraux est associé à une consommation des protons $\mathrm{H}^{+}$dans le milieu réactionnel (Moursalou, 2010). Ce phénomène explique la dissolution du minerai dans les solutions d'acide nitrique au $\mathrm{pH}$ faible. Ce pendant pour les solutions d'acide citrique, il faut ajouter en plus de l'influence des protons $\mathrm{H}^{+}$, le pouvoir chélateur des cations métalliques (ions calcium et fer) par les groupements carboxyliques et hydroxyle. En effet, il a été signalé que la dissolution des phosphates par les acides organiques est due en grande partie au pouvoir complexant des ions métalliques de l'apatite par les groupements carboxyliques et hydroxyles contenus dans ces acides (Agbegnigan, 2013).

\section{Dissolution du minerai par les solutions d'acide sulfurique}

La dissolution du minerai de phosphate naturel par les solutions d'acide sulfurique a été effectuée à $\mathrm{pH}$ inférieur à 4 en raison de la dissolution du minerai en milieu fortement acide. Les résultats obtenus sont indiqués dans la figure 3. On remarque que le taux de phosphore dissous est important avec une valeur de $41 \%$ au $\mathrm{pH}$ inférieur à 2 au bout de 7 heures. Comme dans les autres acides (acide nitrique et citrique étudié ci-dessus) ce taux de dissolution chute au-delà de $\mathrm{pH}$ égal à 2 pour atteindre environ $20 \%$ au tour de $p \mathrm{H}$ égal à 3 . En outre, on constate que ce taux augmente avec le temps de dissolution. Ce qui pourrait être due au fait que l'agitation augmente la surface de contact entre la solution acide et le minerai. 


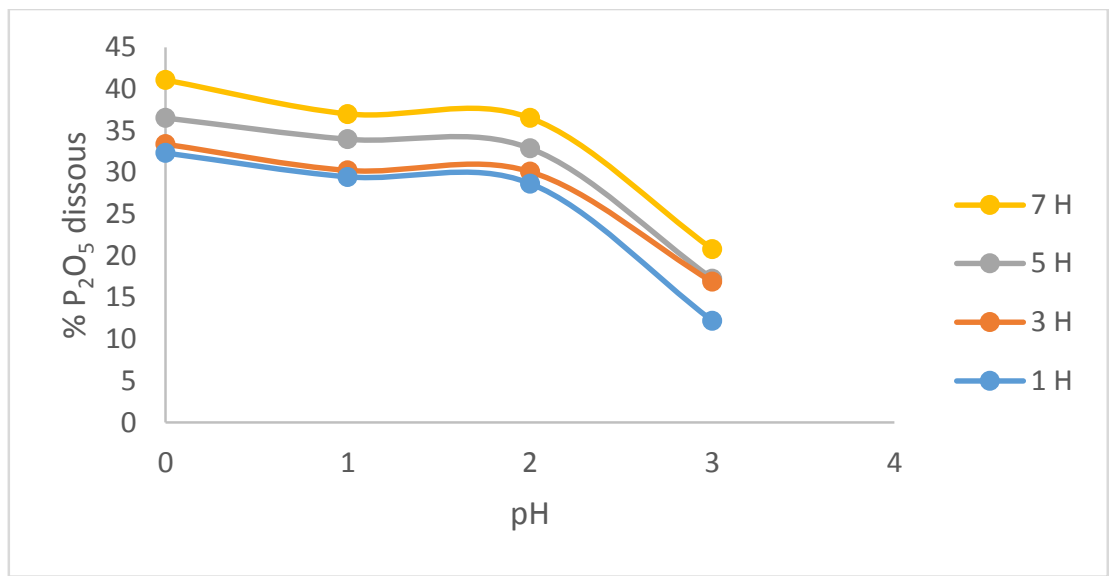

Figure 3 : Taux de phosphore dissous par les solutions d'acide sulfurique en fonction de temps

Etude comparative de la dissolution du minerai par les acides sulfurique, nitrique et citrique

\section{Etude comparative de la cinétique de dissolution du minerai}

L'étude comparative de la cinétique de dissolution du minerai par les acides sulfurique, nitrique et citrique en fonction de la durée d'attaque a été effectuée. Les courbes de dissolution obtenues ont la même allure (Figure 4). On constate que ces courbes présentent une partie raide correspondant à la dissolution rapide du minerai au bout d'une heure d'attaque. Dans cette zone, le taux de phosphore dissous augmente brusquement. Au-delà d'une heure on observe un palier quasi horizontal correspondant à une dissolution lente du minerai. Dans cette zone, très peu de phosphore sont dissous pendant un temps relativement élevé.

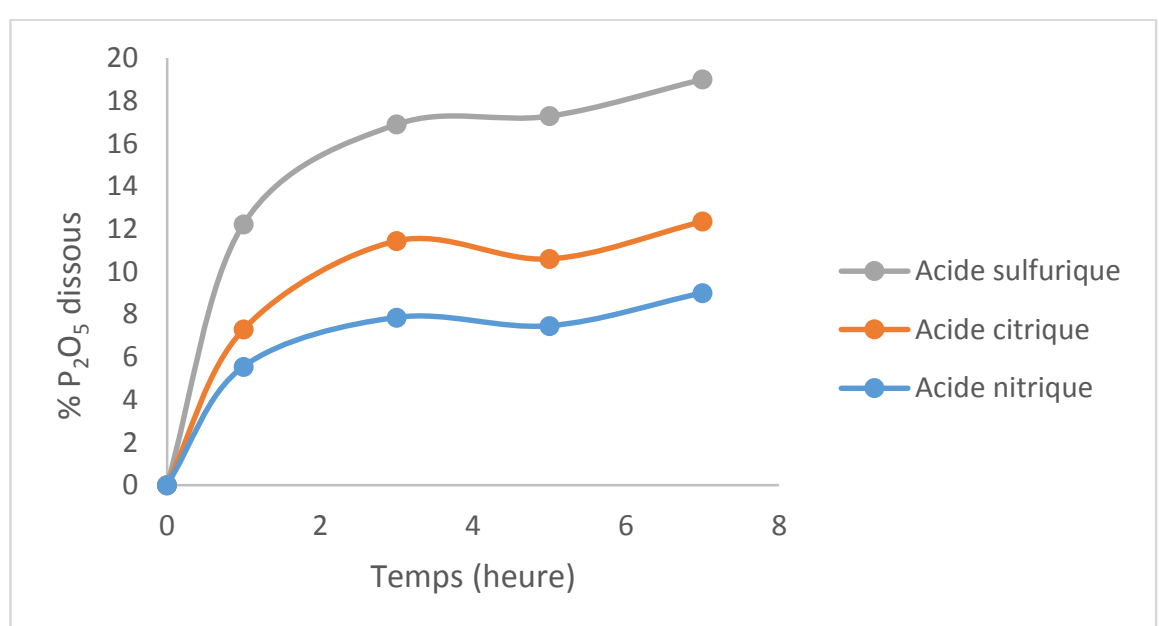

Figure 4 : Comparaison des taux de phosphore dissous par les acides sulfurique, nitrique et citrique à $0,001 \mathrm{~mol} / \mathrm{L}$. 
La première partie des courbes où la dissolution est rapide peut être attribuée à l'efficacité à court terme des phosphates naturels étudiés et la deuxième partie où la dissolution est lente correspond à l'efficacité à long terme. Des résultats semblables ont été obtenus lors de l'étude de la cinétique de dissolution d'une série des phosphates naturels d'Afrique de l'Ouest (Télemsi au Mali, Kodjari au Burkina Faso et Hahotoé au Togo) dans des solutions d'acide citrique à $2 \%$, d'acide formique à $2 \%$ et de citrate d'ammonium (Truong, 1978).

En outre, on constate que quelle que soit la durée d'attaque, l'acide sulfurique dissous mieux le minerai devant l'acide citrique et l'acide nitrique.

\section{Etude comparative de la dissolution du minerai en fonction de la concentration en acide}

Nous avons également réalisé l'étude comparative de la dissolution du minerai en fonction de la concentration des solutions d'acide sulfurique, nitrique et citrique. Les résultats obtenus (Figure 5) montrent que le taux de phosphore dissous augmente lorsqu'on augmente la concentration quelle que soit la solution d'attaque. De plus, l'acide sulfurique dissous mieux le minerai devant l'acide citrique et l'acide nitrique quelle que soit la concentration de la solution d'attaque. Cela pourrait s'expliquer par le fait que l'acide sulfurique étant un diacide, libèrent mieux les protons $\mathrm{H}^{+}$qui interagissent avec les ions phosphates et augmentent en conséquence la dissolution du minerai.

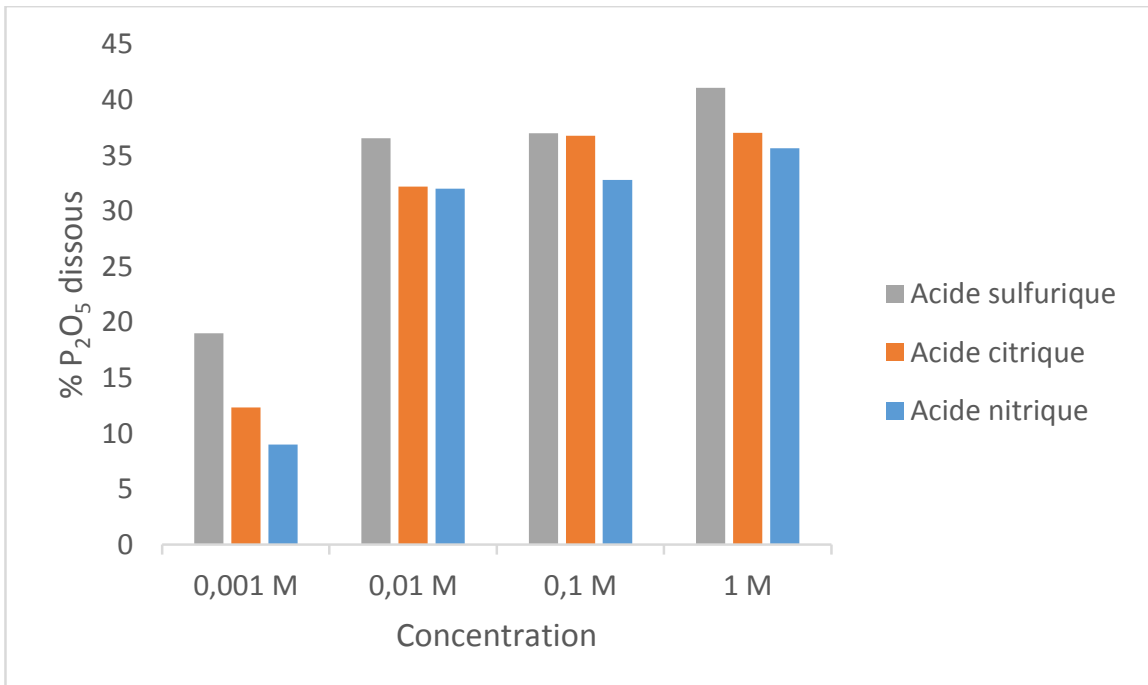

Figure 5 : Comparaison des taux de phosphore dissous par les acides sulfurique, nitrique et citrique pendant 7 heures. 
Le taux maximal de dissolution atteint $41,07 \% ; 37,03 \%$ et $35,64 \%$ respectivement dans les solutions d'acide sulfurique, citrique et nitrique à la concentration de $1 \mathrm{~mol} \cdot \mathrm{L}^{-1}$. Il ressort de ces résultats que le pourcentage de phosphore dissous est resté inférieur à $42 \%$ quelle que soit la concentration de la solution d'attaque utilisée dans cette étude. Cela montre que la dissolution de l'échantillon du phosphate n'est pas totale confirmant ainsi la difficulté de dissoudre complètement certains phosphates naturels [12]. Ce phénomène peut être expliqué par la présence dans le minerai des impuretés comme la silice en quantité non négligeable qui ne favorise pas la dissolution du minerai.

\section{Conclusion}

La dissolution du minerai de phosphates naturels par les solutions d'acides sulfurique, nitrique et citrique a été réalisée. La détermination du taux de phosphore dissous par les différentes solutions d'attaques a été effectuée par le dosage du taux d'anhydride phosphorique $\left(\mathrm{P}_{2} \mathrm{O}_{5}\right)$ dans les filtrats. L'effet du $\mathrm{pH}$ et du temps d'attaque sur la dissolution du minerai ont été mis en évidence. Les résultats de cette étude montrent une solubilité moyenne du minerai dans les solutions fortement acides notamment dans l'acide sulfurique. En milieu faiblement acide, la solubilité est quasi nulle quel que soit le temps d'attaque. L'attaque du minerai de phosphates naturels de Tahoua par les solutions de ces acides minéraux et organique à $\mathrm{pH}$ faible peut ouvrir des voies intéressantes pour l'élaboration des intrants phosphatés hydrosolubles afin de valoriser le produit.

\section{References:}

1. Adel, S. H., Shurooq, T. R., Haider, A. A. (2012). Phosphate Rock Treatment with Hydrochloric Acid for Increasing $\mathrm{P}_{2} \mathrm{O}_{5}$ Content. Engineering and Technology Journal, 30 : 67-76.

2. Agbegnigan, K., Moursalou, K., Tchegueni, S., Koledzi, K. E., Zonvide, E, Dihéénane, D. B., Tchangbedji, G., Mohamed, H. (2013). Étude de la compétitivité de complexation des métaux issus des phosphates naturels de Hahotoé-Kpogamé par les substances humiques. Afrique Science, 09 : 23-33.

3. André, B., Saidou, K., Elias, A. (1998). Fertilité des sols et environnement. Cahier d'études et de recherches francophones/Agriculture, $7: 365$.

4. Eremia-Georgescu, G. (2004). Ajout d'une charge minérale et création d'une porosité dans les ciments apatitiques à usage biologique. Thèse de doctorat. Institut National Polytechnique de Toulouse. 
5. Lukas, B., Dana, R., Ales, H. (2008). Kinetics of dissolution of calcium phosphate (Ca-P) bioceramics. Processing and Application of Ceramics, 2 : 57-62.

6. FAO. (2004). Caractérisation et essais de solubilité des phosphates naturels. Rome : Edition de F Zapata et R.N Roy.

7. Mathieu, C., Piettain, F. (2003). Analyse chimique des sols: méthodes choisies. Paris : Edition Lavoisier/Tec \& Doc.

8. Moursalou, K. (2010). Extraction, caractérisation, réactivité chimique des substances humiques naturelles avec les phosphates de HahotoéKpogamé et leurs propriétés photodégradante. Thèse de doctorat. Université de Lomé.

9. Moursalou, K., Zanguina, A., Tchangbedji, G., Mohamed, E.M., Natatou, I., Mohamed, H., Mohamed, S. (2010). Etude de la dissolution des phosphates naturels de Hahotoé-Kpogamé (Togo) par les acides humiques naturels. Journal de la Société Ouest-Africaine de Chimie, 030 : 41-47.

10. Moustapha, A., Mahaman, L., Zeinabou, H., Attikou, A., Daouda, S. (2003). Lutte contre la désertification au sahel.Rapport d'activités 2001-2002, Projet Régional AIEA, CT RAF/05/48, Niamey, Niger.

11. Natatou, I., Almou, M., Zanguina, A., Ikhiri, K., Boss, A., Bougard, M. (2004). Etude physico-chimique du phosphate marchand de Tahoua (Niger). Journal de la Société Ouest-Africaine de Chimie, $018: 137-148$.

12. Natatou, I., Zanguina, A., Ikhiri, K., Boos, A., Guille, J., Rastegar, F., Burgard, M. (2005). Caractérisation physico-chimique du phosphate naturel de Tahoua (Niger). Annales de Chimie-Sciences des Matariaux, $30:$ 67-76.

13. Truong, B., Pichot, J., Beunard, P. (1978). Caractérisation et comparaison des phosphates naturels tricalciques d'Afrique de l'Ouest en vue de leur utilisation directe en agriculture. Agronomia Tropical, $33: 136-145$.

14. Zanguina, A. (2011). Contribution à la caractérisation physicochimique et à la mise en valeur agronomique du phosphate naturel de Tahoua (Niger) : essais de fabrication de phopho-compostage. Thèse de doctorat, Université de Niamey. 